\section{Teoría Internacional: El caso de un enfoque clásico}

\section{Hedley BulL*}

I

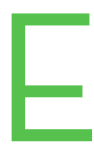
n la actualidad ${ }^{1}$, dos enfoques de la teoría internacional llaman nuestra atención. Al primero de estos lo denominaría el enfoque clásico. Con esto no hago referencia a la crítica y estudio de los "clásicos" de la Relaciones Internacionales, esto es, los escritos de Hobbes, Grocio, Kant y otros grandes pensadores del pasado que dirigieron su atención a los asuntos internacionales. Ciertamente, este estudio ejemplifica el enfoque clásico y provee un método particularmente fructífero e importante. Lo que tengo en mente, sin embargo, es algo mucho más amplio que esto. Se trataría de un enfoque que teorice desde la filosofía, la historia y el derecho, y que esté caracterizado sobre todo por una explícita dependencia en cuanto al ejercicio del juicio y por la asunción de que si nos limitamos a estrictos estándares de verificación y de prueba quedan muy pocas cuestiones relevantes que puedan ser dichas acerca de las Relaciones Internacionales. Se trataría también de que las proposiciones generales sobre esta materia deben por tanto provenir de un proceso de percepción o intuición científicamente imperfecto, y que estas proposiciones generales no pueden ser acordadas más que con una apropiada condición, tentativa e inconclusa, de su incierto origen.

Hasta épocas recientes, virtualmente todos los intentos de teorización sobre las Relaciones Internacionales estaban fundados en el enfoque que acabo de describir. Desde luego, esto lo podemos identificar en la variada sistematización de la teoría internacional del siglo XX -en trabajos como los de Alfred Zimmern, E.H. Carr, Hans Morgenthau, Georg Schwarzenberger, Raymond Aron y Martin Wight-. Este es también el método de varios de sus precursores, de quienes esparcieron ideas y enfoques parciales que ellos habían buscado agrupar: filósofos políticos como Maquiavelo o Burke, juristas internacionalistas como Vattel y Opphenheim, panfleteros como Gentz y Cobben, historiadores como Herren y Ranke. Esta es la razón por la cual este enfoque ha sido durante tanto tiempo el estándar que podemos denominar como clásico.

El segundo enfoque es el que denominaría como científico. He decidido llamarlo científico en vez de cientificista para no prejuzgar la cuestión que querría discutir recurriendo a un término oprobioso. Al usar este nombre para el segundo enfoque, sin embargo, son las aspiraciones de aquellos que lo adoptan lo que ten-
*Hedley BULL

(1932-1985) fue uno de los académicos de las Relaciones Internacionales más importantes del siglo XX. Fue profesor en la Australian National University, en la London School of Economics y en la Universidad de Oxford. Entre sus obras traducidas al español se encuentra La sociedad anárquica. Un estudio sobre el orden en la política mundial, Los libros de la Catarata, Madrid, 2005 [1977]. [Traduccción de Irene Martín Cortés].

La versión original de este artículo fue publicada como Bull, Hedley, "International Theory: The Case for en World Politics, vol. 18 , no. 3 , 1966, pp. 361-377. Copyright Trustees of Princeton University. Publicado por Cambridge University Press. Permisos de reproducción otorgados.

Traducción: Melody FONSECA y Marcelo LUZZI.

\section{DOI:}

http://dx.doi.org/ 10.15366/relacionesinternacionales2018.38.009 a Classical Approach"

1 N.d.T.: "En la actualidad" se refiere a 1966, momento de la publicación original de este artículo. 
go en mente en vez de su desempeño. Ellos aspiran a una teoría de las Relaciones Internacionales cuyas proposiciones estén basadas tanto sobre pruebas lógicas o matemáticas como sobre estrictos y empíricos procedimientos de verificación. Algunos de ellos desestiman las clásicas teorías de Relaciones Internacionales como inútiles, y claramente se conciben a sí mismos como fundadores de una completa ciencia nueva. Otros admiten que los productos del enfoque clásico fueron mejores que nada y, quizás, incluso la miran con cierto aprecio, como un propietario de un modelo de 1965 pudiera mirar el motor vintage de un coche. Pero en ningún caso esperan y creen que su propio tipo de teoría llegará a reemplazar completamente al modelo más antiguo -como los positivistas lógicos cuando desearon apropiarse de la filosofía inglesa de los años treinta o como los "Whiz Kids"2 del señor McNamara cuando se mudaron al Pentágono, se vieron a sí mismos como tenaces y expertos nuevos hombres, encargándose de una decadente y lanuda disciplina, o pseudo disciplina-, que hasta ahora ha sido gestionado por alguna extraña rareza para evitar el método científico, pero que finalmente siempre ha cedido.

El enfoque científico a la teoría de las Relaciones Internacionales, así definido, está presente en la Teoría de los Sistemas Internacionales, como los desarrolló Morton A. Kaplan y otros, en las diversas extrapolaciones internacionales de la Teoría de Juegos de John Von Neumann y Oskar Morgenstern, en la Teoría de Negociaciones de Thomas C. Schelling, en los trabajos de comunicación social de Karl W. Deustch, en el estudio de alianzas políticas de William H. Riker, en el modelo de foreing policy-making producido por George A. Modelski y otros, en el estudio matemático de las carreras de armas y las peleas mortales de Lewis $\mathrm{F}$. Richardson, así como en las teorías del conflicto desarrolladas por Kenneth Boulding y Anatol Rapoport. Esto también parece ser una parte importante del contenido de la llamada "investigación para la paz"3.

Los estudios que he nombrado varían enormemente en los métodos que emplean y en las cuestiones que dirigen. Sus autores, en efecto, lejos de enfrentar el mundo exterior como un frente común, mayoritariamente se miran unos a otros con la hostilidad de los líderes de la secta marxista. Se debe plantear que también existen importantes discrepancias entre ellos en la medida en que han iluminado nuestro tema. Lo que he denominado enfoque científico, además, no está presente en el mismo grado en todos ellos. Se corre el riesgo de agruparlos a todos juntos, y puede ser inevitable que las críticas dirigidas al conjunto del género sean injustas a algunas partes del mismo. Aun así, todos estos estudios y modas personifican el enfoque científico en alguna medida, y para discutirlo es necesario limitar nuestra atención

2 N.d.T.: Los "Whiz Kids" de Robert McNamara era el nombre con el que la prensa llamaba al grupo de expertos de la RAND Corporation que trabajaban a cargo del Secretario de Defensa norteamericano McNamara, especialmente en asuntos de análisis económico y sistemas de gestión.

3 Véanse, por ejemplo, Kaplan, Morton A., System and Process in International Politics, John Wiley \& Sons, Nueva York, 1957; Morgenstern, Oskar, The Question of National Defense, Random House, Nueva York, 1959; Schelling, Thomas, The Strategy of Conflict, Harvard University Press, Cambridge, 1960; Deutsch, Karl, et al., Political Community and the North Atlantic Area: International Organization in the Light of Historical Experience, Princeton University Press, Princeton, 1957; Riker, William, The Theory of Political Coalitions, Yale University Press, New Haven, 1962; Modelski, George, A Theory of Foreign Policy, Praeger for the Center of International Studies, Princeton University, Nueva York, 1962; Richardson, Lewis, Arms and Insecurity: A Mathematical Study of the Causes and Origin of War, Boxwood Press, Pittsburg, 1960; y Wright, Quincy y C.C. Lienau (eds.), Statistics of Deadly Quarrels, Boxwood Press, Pittsburg, 1960; Boulding, Kenneth, Conflict and Defense: A General Theory, Harper \& Brothers, Nueva York, 1962; Rapoport, Anatol, Fights, Games, and Debates, University of Michigan Press, Ann Arbor, 1960. 
en lo que tienen en común.

En Estados Unidos, en los últimos diez años el enfoque científico ha progresado de ser una actividad marginal en los estudios académicos de las Relaciones Internacionales hasta tal posición que, al menos, es posible argumentar que ha devenido en la metodología ortodoxa en la materia. En 1963, la entrega del premio de la Asociación Americana de Ciencias Políticas para el mejor estudio del año para un practicante del enfoque clásico (para Inis Claude por su Power an International Relations) tuvo siempre la apariencia de una acción perversa de la retaguardia.

En la comunidad académica británica, por el contrario, el enfoque científico en las Relaciones Internacionales prácticamente no tuvo impacto. El único inglés que ha hecho la mayor contribución en el nuevo género - Lewis F. Richardson- trabaja solo y sin ser reconocido, y cuando hace unos años su trabajo fue exhumado y aclamado como el de un gran pionero, fue hecho por editores estadounidenses quienes se dirigían a un público predominantemente norteamericano. No solamente los estudiantes británicos de Relaciones Internacionales no han buscado contribuir a la teoría desde este enfoque, pero, con una o dos excepciones, el trabajo de los norteamericanos y otros autores que han arado este campo ha fallado en atraer su respeto o, incluso, su atención.

Si estaba claro que este desdén ha sido fundado sobre una comprensión del enfoque científico y en un considerado rechazo de este, podría no haber causa para que nosotros revisásemos nuestra actitud. Podemos incluso ver en nuestra impermeabilidad a esta moda, la prueba de la fundamental solidez y firmeza de nuestro propio enfoque. La actual posición, sin embargo, es que somos en gran medida ignorantes de lo que la nueva literatura contiene y que nuestro rechazo no se deriva tanto de una crítica razonada como de sentimientos de repulsión estética contra su lenguaje y método, irritación con sus a veces arrogantes y ridículas afirmaciones, frustración con nuestra incapacidad para captar sus significados o emplear sus herramientas, en la a priori confianza en que como empresa intelectual está obligada a fracasar, e inseguridad profesional inducida por el horrible e insistente pensamiento de que quizás pueda tener éxito.

No hay duda de que el estilo que ha emergido desde el enfoque científico debería considerarse seriamente. Juzgado por sus propios estándares de precisión lógica y rigor científico, su cualidad es a veces alta. Además, aunque contrario a la opinión que tenemos de esta literatura, resulta imposible examinarla con algún grado de cuidado y simpatía y también concluir que su contribución a la comprensión de las Relaciones Internacionales es nula. En efecto, tomando la gran concentración de energía y talento que ha ido produciéndose en los años recientes, sería extraordinario si esto fuese diferente.

Resulta deseable, por lo tanto, que si vamos a rechazar el enfoque científico, al mismo tiempo debemos prestarle atención y formular algunas objeciones tal como haremos. Se ha desarrollado ahora tanto ímpetu que su silenciamiento, o peor, el superficial abuso con que a veces es recibido por los críticos británicos, ya no será suficiente para mantenerlo a raya. Si, como creo, el enfoque científico debiera mantenerse en el segundo plano, solo podría conseguirse por la crítica racional. 


\section{II}

Pretendiendo proveer una crítica racional, uno puede comenzar por descartar un número de reclamos normalmente dirigidos al enfoque científico que están al lado del punto. Uno de esos reclamos formulados por estos teóricos, especialmente, quizás, de Morton A. Kaplan, es que su escritura es tortuosa y poco elegante. Pero el hecho de que el libro de Morton A. Kaplan no sea un placer para leer no es una crítica a la teoría de la política internacional, como tampoco lo es que la dificultad de leer a Einstein sea una deficiencia de la Teoría de la Relatividad. Si Kaplan pudiera ser acusado de construir deliberadamente una terminología innecesariamente oscura, o de emplearla torpe e ineficientemente, sería otra cuestión; pero tal acusación estaría bastante fuera de lugar. La terminología de Kaplan resulta ser una parte vital de su completo intento de construir un sistema riguroso, y su uso es preciso y económico.

En efecto, mientras uno no necesita ir tan lejos como considerar la mediocridad literaria como un mérito positivo en un libro sobre política, gran parte de la originalidad y fuerza del trabajo de Kaplan deriva precisamente de este desdén por la tradición que considera a los textos históricos y políticos como una rama de las belles lettres. El poder de esta tradición refleja el hecho de que los textos históricos y políticos, además de servir de finalidad de comunicación entre especialistas buscando comprender el tema, sirven para otros objetivos como educación, persuasión, entretenimiento público, y la exhibición de logros caballerescos. Kaplan es verdaderamente correcto en desestimar la literatura de ornamentación que es un elemento propio en los textos de esta última finalidad como una irrelevancia y obstáculo en los textos anteriores.

Otra insatisfactoria línea de crítica es la que no se centra en la doctrina de los teóricos científicos pero sí en los motivos que los conducen a proponerla. Por consiguiente, se ha observado que quienes siguen el enfoque científico son nuevos escolásticos, quienes desean refugio en un mundo esencialmente de construcciones intelectuales para escapar de la realidad política; que ellos son los científicos naturales, matemáticos, y economistas manqués que, incapaces de hacer carrera por sí mismos en sus propios campos, se han movido a otro donde la situación es más fácil, llevándose consigo sus técnicas; que ellos están interesados en elaborar una metodología matemática o científica por su propio beneficio -o por el beneficio de demostrar su maestría a los no familiarizados- más que en esclarecer nuestro tema usando esta metodología; o incluso que ellos representan una nueva forma de culto cargo.

Estas observaciones, o algunas de ellas, son verdad o medias verdades, y nos ayudan a entender el carácter de la nueva teoría como un movimiento intelectual. Esto es cierto de cualquier estilo intelectual o moda académica que es perseguido por una variedad de motivos de los cuales el desinteresado deseo de conocimiento es solo uno de ellos, y que algunos de estos motivos son separados de cualquier deseo e incluso son desacreditados. Pero precisamente por esta razón, una discusión de las motivaciones de los teóricos no provee ninguna base para la defensa de un estilo intelectual contra otro. Es muy sencillo para el teórico de mentalidad científica dar vuelta a la tortilla. ¿Acaso aquellos que se adhieren al enfoque clásico lo hacen por el interés conferido en sus propias técnicas, una vaga reticencia a aprender nuevas? ¿Acaso no están casados con una metodología por su propio bien, al arte de juzgar por encima y contra la medición, y a formas literarias contrarias a lo simbólico, aferrándose 
a estos instrumentos de su oficio como caballería en la era de la mecanización? ¿Acaso no representan una extrovertida generación preparada en un conjunto de técnicas, expresando su animadversión contra una generación entrante formada en otras técnicas? Debería estar más seguro de lo que estoy de que mis propios motivos en la preparación de este escrito son completamente imparciales antes de invitar a la crítica de los demás atacando la de los otros. Estaremos bien asesorados, por lo tanto, para limitar nuestra atención a las propias doctrinas.

Por último, es un error ver en el enfoque científico, o en cualquiera de los métodos que se inventen, el instrumento de cualquier finalidad política en la política exterior o de defensa. En el nivel de los teóricos de sistemas, de los teóricos del juego, de los teóricos de la comunicación, y de los teóricos del conflicto, es posible encontrar actitudes que oscilan desde el más conservador hasta el más radical. Tampoco hay ninguna conexión lógica entre alguna de las técnicas y alguna actitud política particular. Escritores como Herman Kahn, Thomas Schelling, y Morton Kaplan, a quienes se puede describir ampliamente como pro establishment en sus actitudes hacia la política exterior y de defensa, han sido objeto de ataques políticos que giran sobre su uso de estas técnicas. Pero ningún ataque toma en cuenta otros autores como Kenneth Boulding, Anatol Raport o J. David Singer, quienes son disidentes de la política exterior y de defensa de Estados Unidos aunque sean intelectuales en el mismo campo. De manera similar, la actual tendencia de la "investigación para la paz" o de "resolución de conflictos" a menudo parece reflejar la equivocada idea de que la aplicación de estas nuevas técnicas al estudio de las Relaciones Internacionales obliga a reivindicar políticas radicales o facilita su implementación.

De todas formas, el enfoque científico ha contribuido y es probable que contribuya muy poco a la teoría de las Relaciones Internacionales, y en la medida en que tiene la intención de invadir y, en última instancia, desplazar el enfoque clásico, es sumamente perjudicial. En favor de esta conclusión, deseo ofrecer siete propuestas.

La primera propuesta es que al ellos mismos limitarse a lo que pueda ser lógicamente o matemáticamente probado o verificado de acuerdo con procedimientos estrictos, los practicantes del enfoque científico están negando los únicos instrumentos que están actualmente disponibles para abordar la sustancia del tema. Al abstenerse de lo que Morton Kaplan llama "suposiciones intuitivas" o lo que William Riker denomina "literatura sabia", se están comprometiendo con un rumbo de puritanismo intelectual que los mantiene (o los mantendría si realmente se adhieren) tan alejados de la sustancia de la política internacional como los reclusos de un convento victoriano estaban del estudio del sexo.

Para apreciar nuestra confianza sobre la capacidad de juicio en la teoría de las Relaciones Internacionales, solamente tenemos que ensayar algunas de las cuestiones centrales a las que se dirige esa teoría. Algunas de estas son, al menos en parte, cuestiones morales, que por su propia naturaleza no pueden recibir una respuesta objetiva, y que solo pueden ser indagadas, esclarecidas, reformuladas y provisionalmente respondidas desde una postura arbitraria, acorde al método filosófico. Otras de estas son las preguntas empíricas, pero de naturaleza tan imprecisa que cualquier respuesta que les proporcionemos dejará algunas cosas sin decir, no serán más que un punto de una conversación que aún no se ha concluido. No es simplemente que al encuadrar las hipótesis en función a estas preguntas empíricas de- 
pendemos de la intuición o el juicio (como a menudo ha sido señalado, esto es tan cierto en las ciencias naturales como en las sociales); es que en la prueba de ellas también dependemos completamente del juicio, sobre una áspera y dispuesta observación, de una clase para la cual no hay espacio en la ciencia lógica o estricta, que las cosas son y no son así.

Por ejemplo, ¿constituye el conjunto de estados soberanos una sociedad o sistema político, o no? Si podemos hablar de una sociedad de estados soberanos, ¿presupone esto una cultura o civilización común? Y si esto es así, ¿es este tipo de cultura común la base de una red diplomática mundial en la que ahora intentamos intervenir? ¿Cuál es el lugar de la guerra en la sociedad internacional? ¿Todo uso privado de la fuerza es un anatema para el funcionamiento de la sociedad, o hay guerras justas que se pueden tolerar o, incluso, requerir? ¿Un estado miembro de la sociedad internacional disfruta del derecho a intervenir en los asuntos internos de otro estado, y bajo qué circunstancias? ¿Son los estados soberanos los únicos miembros de la sociedad internacional, o en última instancia consisten en seres humanos individuales, cuyos derechos y deberes anulan a los de las entidades que actúan en su nombre? ¿En qué medida el curso de los eventos diplomáticos está determinado o circunscrito en un momento dado por la forma general o la estructura del sistema internacional? ¿Por el número, peso relativo y la disposición radical o conservadora de los estados miembros? ¿Y por los instrumentos que la tecnología militar o la distribución de la riqueza ha puesto en sus manos para conseguir sus objetivos, así como por el conjunto particular de reglas del juego que subyace a la práctica diplomática en cada momento? Y etcétera.

Estas son las típicas preguntas que componen, esencialmente, la teoría de las Relaciones Internacionales. No obstante, los teóricos científicos han renunciado a los medios para entenderse directamente con ellos. Cuando se los confronta, hacen una de estas dos cosas: o se asustan y se dedican a temas periféricos - metodologías para acordar con el tema, extrapolaciones lógicas de marcos conceptuales para pensarlo, notas al margen del tema que sean susceptibles de medida o de observación directa - o se liberan de su propio código y recurren de repente y sin reconocer que esto es lo que están haciendo con los métodos del enfoque clásico - métodos que en algunos casos emplean muy mal, sus preocupaciones y entrenamientos dejándolos extraños a la sustancia del tema-.

La incapacidad congénita del enfoque científico para tratar con el quid de la cuestión mientras se mantiene fiel a sus propios términos, me lleva a una observación sobre la enseñanza de la asignatura en las universidades. Cualesquiera sean las virtudes que uno pueda discernir en el enfoque científico, es un desarrollo totalmente retrógrado que ahora conformará la base de los cursos de enseñanza de grado en política internacional, como sucede en algunas universidades de Estados Unidos. El estudiante cuyos estudios de política internacional consisten únicamente en una introducción de las técnicas de la teoría de sistemas, de la teoría de juegos, de la simulación, o del análisis de contenido, simplemente se desconecta de la materia y es incapaz de desarrollar cualquier sensibilidad tanto sea por el juego de las políticas internacionales como por los dilemas morales a los que da lugar.

La segunda propuesta que deseo proponer surge de la primera: es que donde los practicantes del enfoque científico han logrado arrojar luz sobre la sustancia del tema, ha sido en el ir más allá de los límites de ese enfoque y al emplear el método clásico. Lo que es un valor 
en sus trabajos consiste básicamente en juicios que no han sido establecidos por los métodos matemáticos o científicos que emplean, y a los que han llegado independientemente de ellos.

Permítanme tomar como ejemplo el trabajo de Thomas Schelling, quien ha contribuido tanto como, y quizás más que, cualquier otro pensador del género científico a la teoría de las Relaciones Internacionales. Su elaboración de la noción de control de armas, los elementos de disuasión, la naturaleza de las negociaciones, el lugar en las Relaciones Internacionales de los tratados de fuerza tiene una excepcional originalidad e importancia y probablemente demostrarán haber dejado una huella duradera en la teoría y, ciertamente, en la práctica de estos asuntos. Al mismo tiempo, es un economista experimentado: ha escrito estudios de naturaleza técnica acerca de la teoría del juego y negociación, y a veces ha parecido prestar su apoyo a la convocatoria para más teoría que la de un tipo científico.

Parece que las reveladoras observaciones de Schelling acerca de la violencia y la política internacional en cualquier caso tienen el estatus de juicios indemostrables e improbables, y que no han sido y no han podido ser demostradas por su trabajo en la teoría formal del juego y la negociación. Schelling combina su interés en las últimas técnicas con un astuto juicio político y una habilidad filosófica para pensar los problemas en términos de sus elementos básicos. Es posible que sus ideas acerca de las Relaciones Internacionales las haya sugerido por sus estudios técnicos, y evidentemente lo ha considerado útil para proporcionar ilustraciones de sus ideas en ejercicios formales y teóricos. Aquellos de sus lectores que comparten su interés en estas técnicas lo encontrarán divertido $y$, tal vez, rentable dedicarse a estas ilustraciones. Pero en el mejor de los casos son una analogía útil: no representan los cimientos de su contribución a la política internacional o el camino que debe seguirse para llegar a ello.

Mi tercera propuesta es que es poco probable que los practicantes del enfoque científico progresen de la forma que aspiran. Algunos de los autores que he discutido estarían suficientemente preparados para admitir que hasta ahora solo se han tratado temas periféricos de una manera rígidamente científica. Pero su reclamo sería que no es por el desempeño hecho hasta ahora que su enfoque debe ser juzgado, sino por la promesa que contiene de último avance. Incluso dicen que la modestia de sus inicios pone en evidencia cuán fieles son al ejemplo de las ciencias naturales: la física moderna también, Morton A. Kaplan nos dice, "ha erigido su alto presente al plantearse problemas para los que tiene las herramientas o técnicas para resolver"4.

La esperanza es fundamentalmente que nuestro conocimiento de las Relaciones Internacionales alcance el punto en el que se vuelva genuinamente acumulativo: que desde el presente, un sinfín de terminologías y marcos conceptuales que compiten entre sí surgirá un lenguaje común, que la insignificante variedad de temas que ahora ha sido científicamente representados eventualmente se unirán y se volverán importantes, y que existirá entonces una base de teoría firme sobre la cual los recién llegados a la empresa edificarán.

\footnotetext{
4 Kaplan, Morton A. "Problems of Theory Building and Theory Confirmation in International Politics" en World Politics, vol. 14, no. 1, 1961, p. 7.
} 
Nadie puede asegurar que esto no sucederá, pero las perspectivas ciertamente son muy desoladoras. Las dificultades que la teoría científica ha encontrado no parecen surgir de la cualidad que se supone que las Relaciones Internacionales tiene de "atrasada" o ciencia olvidada, sino de las características inherentes a la materia que han sido catalogadas con la suficiente frecuencia: el incontrolable número de variables que cualquier generalización acerca del comportamiento del estado debe ser tenido en cuenta; la resistencia del material al experimento controlado; la cualidad que tiene de cambiar ante nuestros ojos y deslizarse entre nuestros dedos, incluso cuando tratamos de categorizarlo; el hecho de que las teorías que producimos y los casos que están teorizados están relacionados no solo como sujeto y objeto sino también como causa y efecto, garantizando así que incluso nuestras ideas más inocentes contribuyan a su propia verificación o falsificación.

Un futuro más probable para la teoría de política internacional es que permanecerá indefinidamente en la etapa filosófica del constante debate sobre los fundamentos; que los trabajos de los nuevos teóricos científicos no probarán ser una sólida subestructura en la que la nueva generación edificará, sino más bien que aquellos de ellos que sobrevivan tomarán su lugar junto a trabajos anteriores como guías parciales e inciertas de un tema esencialmente intratable; y que los sucesivos pensadores, mientras aprenden lo que pueden de lo que ha pasado antes, se seguirán sintiendo impelidos a edificar sus propias casas de teorías desde los cimientos.

Una cuarta propuesta que se puede adelantar contra muchos de los que pertenecen a la escuela científica es que han hecho un gran perjuicio a la teoría en este campo al concebirla como la construcción y manipulación de los llamados "modelos". La investigación teórica de un tema empírico normalmente procede por medio de la afirmación de conexiones y distinciones generales entre eventos en el mundo real. Pero es la práctica de muchos de estos escritores la que proyecta sus teorías en la forma de una abstracción deliberadamente simplificada de la realidad, que luego vuelcan y examinan de una u otra manera antes de considerar qué modificaciones deben efectuarse si se van a aplicar en el mundo real. Un modelo, en sentido estricto, es un sistema deductivo de axiomas y teoremas; tan común se ha vuelto el término que, incluso, se usa también para referirse a lo que simplemente es una metáfora o una analogía. Solo es la técnica de construir modelos en sentido estricto lo que aquí está en cuestión. No importa cuán valiosa haya sido esta técnica en economía y otros temas, su uso en política internacional es deplorable.

La virtud que se supone que reside en los modelos es que al liberarnos de la restricción de la referencia constante a la realidad, nos dejan libres para establecer simples axiomas basados en algunas variables y de ahí en adelante limitarnos a una lógica deductiva rigurosa, generando de este modo amplias perspectivas teóricas que proporcionarán una amplia base de datos para guiarnos en el mundo real, incluso si no cubre los detalles.

No conozco ningún modelo que haya ayudado a nuestra comprensión de las Relaciones Internacionales que no habría podido ser expresado como una generalización empírica. Esto, sin embargo, no es la razón por la cual debemos abstenernos de ellos. La libertad de mirar el mundo que tiene el constructor de modelos de la disciplina, es lo que lo hace peligroso; se desliza fácilmente en un dogmatismo que la generalización empírica no permite, 
atribuyendo al modelo una conexión con la realidad que no tiene, y con la frecuencia de no distorsionar el modelo mismo importando suposiciones adicionales sobre el mundo bajo el disfraz de axiomas lógicos. La completitud intelectual y el orden lógico de la operación de construcción de modelos le da un aire de autoridad que a menudo es bastante engañoso en cuanto a su posición como una declaración sobre el mundo real.

Tomaré como un ejemplo al más ambicioso de todos los constructores de modelos: Morton Kaplan. Este nos provee dos modelos históricos y cuatro posibles sistemas internacionales, cada uno con sus "reglas esenciales" o comportamiento característico. Afirma que el modelo le permite hacer predicciones - solo es cierto con un alto nivel de generalizaciónacerca de las características o del comportamiento modal en el presente sistema internacional, sobre si es probable o no la transformación de este sistema en otro y qué forma podrían tomar.

Los seis sistemas que Kaplan identifica, y las "reglas esenciales" o características de comportamiento de cada uno de ellos, son, de hecho, ideas bastante comunes, extraídas de la discusión cotidiana de los asuntos internacionales, sobre la estructura política general que el mundo ha tenido o podría tener. Son los sistemas políticos internacionales del siglo XVIII y XIX, el actual sistema llamado bipolar, la estructura que podría existir si la presente polarización de poder no fuese moderada por las Naciones Unidas y por poderosos terceros partidos, el sistema que podríamos tener si las Naciones Unidas se convirtieran en la fuerza política predominante en un mundo de estados todavía soberanos, un estado mundial y un mundo de muchas potencias nucleares.

Discutiendo las condiciones bajo las cuales el equilibrio se mantiene en cada uno de estos sistemas, y prediciendo la probabilidad y la dirección de su transformación en diferentes sistemas, Kaplan parece recurrir a una suerte de conjetura mucho más arbitraria que cualquiera de los involucrados en el estilo de la teoría internacional que desea desplazar. Discutiendo los dos sistemas históricos, Kaplan usa algunos ejemplos pertinentes desde la historia reciente, pero no hay razón para asumir que el comportamiento en el futuro sistema internacional de este tipo será el mismo. En su discusión de los sistemas no históricos, sus observaciones o bien son extensiones tautológicas de las definiciones que emplea, o son juicios empíricos formulados arbitrariamente que no pertenecen en absoluto propiamente al modelo.

Los seis sistemas de Kaplan no son, por supuesto, los únicos posibles. Admite, por ejemplo, que no cubren los casos de la antigua Grecia o de la Edad Media, y no abarcan la infinita variedad que el futuro podría develar. ¿Qué razón hay, por lo tanto, para suponer que la transformación de cualquiera de los sistema deba estar en uno de los otros? Toda la empresa de intentar predecir la transformación sobre la base de estos modelos requiere todas las etapas en que nos encontramos fuera de los modelos e introducir consideraciones adicionales.

Una objeción a los modelos de Kaplan, por tanto, es que en realidad no son modelos: carecen de rigor interno y de consistencia. Pero incluso si poseyeran tales cualidades, no proporcionarían la iluminación de la realidad que Kaplan reclama para ellos. No tenemos forma de saber que las variables excluidas de los modelos no serán cruciales. Kaplan ha pro- 
porcionado un ejercicio intelectual y no más. No quisiera afirmar que alguien que explore la cuestión de qué cambios podrían tener lugar en el sistema internacional actual, o la cuestión de cuál podría ser la forma y la estructura de un mundo de muchas potencias nucleares, es incapaz de extraer algunos indicios de valor del trabajo de Kaplan. Pero cuánto más fructífera puede ser esta cuestión explorada, cuanto mejor podría haber sido tan talentosa una persona como el propio Kaplan los ha explorado, prestando atención a la variedad real de eventos en el mundo real, tomando nota de los muchos elementos que están empujando el presente sistema internacional de una manera u otra, y la gran cantidad de factores técnicos políticos que pueden contribuir a moldear un mundo de muchas potencias nucleares en una docena de formas diferentes de las que pueden estar confinados dentro de los límites del modelo de Kaplan.

La moda de construir modelos ejemplifica una tendencia mucho más amplia y más antigua en el estudio de los asuntos sociales: la sustitución de herramientas metodológicas y la pregunta "¿son útiles o no?" para la aseveración de proposiciones sobre el mundo y la pregunta "¿son verdad o no?". Aunque aparentemente endémico en el pensamiento reciente, creo que este cambio ha sido para peor. La "utilidad" de una herramienta tiene que traducirse finalmente como la verdad de una proposición, o una serie de proposiciones, avanzadas sobre el mundo, y el efecto de la sustitución es simplemente oscurecer la cuestión de una prueba empírica y allanar el camino para el pensamiento de mala calidad y la subordinación de la investigación a la utilidad práctica. Sin embargo, este es un tema que requiere más desarrollo del que se puede dar aquí, y al presentarlo tal vez estoy asumiendo más antagonistas de los que necesito para mi propósito actual.

Una quinta propuesta es que el trabajo de la escuela científica está, en algunos casos, distorsionado y empobrecido por el fetiche por la medición. Para cualquiera que se dedique a la precisión científica, la cuantificación de los tema debe aparecer como el ideal supremo, si toma la forma de la expresión de las teorías mismas en forma de ecuaciones matemáticas o simplemente la de la presentación de evidencia acumulada en forma cuantitativa. Al igual que el obispo anglicano que hace aproximadamente un año comenzó su sermón sobre la moral diciendo que no creía que todas las relaciones sexuales fueran necesariamente incorrectas, deseo adoptar una visión liberal de este asunto. No hay nada inherentemente objetable, así como no hay nada lógicamente peculiar, en una declaración teórica sobre la política internacional moldeada en forma matemática. Tampoco hay ninguna objeción al recuento de fenómenos que no difieran unos de otros en ningún aspecto relevante, y que lo presente como evidencia en apoyo de una teoría. La dificultad surge cuando la búsqueda de lo medible nos lleva a ignorar las diferencias relevantes entre los fenómenos que se están contabilizando, a imputar a lo que se ha contado un significado que no tiene, o a distraernos tanto por las posibilidades que abundan en nuestro sujeto a contar como para desviarse de las investigaciones cualitativas que en la mayoría de los casos son más fructíferas.

Quisiera tomar como ejemplo el trabajo de Karl Deustch y su discípulo Bruce Russett. Estos escritores han buscado investigar los lazos de comunidad que unen a diferentes naciones, y al explicar la cohesión o la capacidad de respuesta mutua que existe entre diferentes pueblos o diferentes grupos dentro de un solo pueblo, han centrado especialmente su atención en la comunicación social, es decir, el flujo de personas, bienes e ideas, o de los "men- 
sajes" que llevan. Karl Deutsch, junto con un número de colaboradores, ha proporcionado un estudio de la medida en que los diversos pueblos del área del Atlántico Norte están vinculados por tales vínculos de comunidad, y le preocupa particularmente la cuestión de la medida en que estos pueblos forman lo que él Ilama una "comunidad de seguridad" - es decir, un grupo de personas que están de acuerdo en que sus problemas comunes deben ser resueltos mediante un "cambio pacífico", y que durante mucho tiempo han tenido expectativas confiables de que sus problemas de hecho se resolverían de esta manera- ${ }^{5}$. Bruce Russett ha abordado el tema de la comunidad simplemente en la relación entre Gran Bretaña y Estados Unidos, y ha buscado determinar en particular si estos dos pueblos se han vuelto más o menos "receptivos" entre sí a medida que el siglo XX ha progresado ${ }^{6}$.

Una característica del trabajo de ambos autores es su presentación de material cuantitativo como un índice del grado de comunidad que existe entre un pueblo y otro. Producen cifras, por ejemplo, sobre los recursos dedicados al comercio como una proporción de los recursos totales: correo enviado al exterior, o a un destino particular, como una proporción del correo total; número de acuerdos diplomáticos concertados con otro país como proporción del total de acuerdos alcanzados; intercambio de estudiantes; "análisis de contenido" de periódicos y revistas inclinados, etc.

El trabajo de Karl Deutsch y Bruce Russett en este campo es ciertamente original y sugerente. Es más, estos autores no son acríticos en su uso del análisis cuantitativo. No obstante, la preeminencia que le conceden es una fuente de debilidad más que de solidez en sus argumentos. Su conteo a menudo ignora (o, si no ignora, pasa por alto) las diferencias más relevantes entre las unidades contadas: diferencias entre el contenido de un punto de un correo y otro, la importancia diplomática de un tratado y otro, la importancia de una pulgada de columna de periódico y otro. Las diferencias en estos otros aspectos relevantes pueden anularse, pero también puede que no; y, en la práctica, es probable que respetemos estas estadísticas solo en los casos en que confirmen alguna impresión intuitiva que ya tenemos, como, por ejemplo, donde las cifras de Russett confirman, como muchos de ellos lo hacen, el juicio muy seguro que podemos hacer a medida que avanza este siglo: que Estados Unidos se ha vuelto relativamente más importante para Gran Bretaña que Gran Bretaña para Estados Unidos. Aun así, tal juicio es bastante externo a las estadísticas que se proporcionan, y no establece que midan nada relevante.

Deutsch y Russett, además, se inclinan a atribuir a sus estadísticas un lugar en la cadena de la argumentación que estas no tienen. A menudo parecen suponer que hay un enfrentamiento tan irrefutable y definitivo sobre una prueba que se puede poner en cifras que se absuelven de la necesidad de mostrar en detalle cómo esta respalda la tesis general que intentan demostrar. El comercio exterior es el comercio exterior, y una medición precisa del comercio exterior no es una medida precisa de nada más, a menos que exista una explicación

5 Karl Deutsch, por supuesto, ha sido autor o coautor de un gran número de trabajos además de Political Community and the North Atlantic Area, Princeton University Press, Nueva Jersey, 1957; pero además de su Political Community at the International Level, Princeton University Press, Nueva Jersey, 1953, es este es el que más se enfrenta con la teoría de las Relaciones Internacionales.

6 Russet, Bruce, Community and Contention: Britain and America in the Twentieth Century, Cambridge University Press, Cambridge, 1963. 
de por qué esto es así. Algunos de los eslabones cruciales pero ausentes en la cadena de argumentos de Deutsch parecen haberse perdido de vista debido a esta tendencia de aquellos que han logrado producir cifras para cegarse por la iluminación que emiten. ¿Son las cifras del "flujo de comunicación" un índice de la comunidad política a nivel internacional, o una de sus causas? ¿El "flujo de comunicación" contribuye a producir el elemento vital, en el esquema de Deutsch, de "identificación mutua", o por el contrario, surge de una manera bastante diferente?

Finalmente, incluso si uno puede admitir que las estadísticas tienen lugar en una investigación sobre la comunidad política y la comunicación social, me parece que Deutsch y Russett se han distraído con ellas en las partes más útiles del tema. Por mucho, las cosas más interesantes que estos dos autores tienen que decir radican en sus intentos de pensar en las características distintivas de una comunidad, los diferentes tipos de comunidades que las obtienen, los elementos que componen la cohesión de una comunidad, los determinantes de la mutua capacidad de respuesta entre un pueblo y otro. Y, con mucho, la evidencia más pertinente que aportan radica en los juicios cualitativos que pueden aplicar a la historia y los asuntos contemporáneos.

Mi sexta propuesta es que exista una necesidad de rigor y precisión en la teoría de la política internacional, pero que el tipo de rigor y precisión que admite el tema puede acomodarse fácilmente dentro del enfoque clásico. Algunos de los objetivos a los que los teóricos científicos apuntan sus observaciones críticas son bastante legítimos. La teoría clásica de las Relaciones Internacionales a menudo ha estado marcada por la incapacidad de definir términos, observar cánones de procedimiento lógicos o hacer suposiciones explícitas. También a veces, especialmente cuando se asoció con la filosofía de la historia, intentó perseguir las implicaciones políticas internacionales de una visión del mundo fundamentalmente no científica. La teoría de las Relaciones Internacionales debería, sin duda, intentar ser científica en el sentido de ser un cuerpo de conocimiento coherente, preciso y ordenado, y en el sentido de ser consistente con los fundamentos filosóficos de la ciencia moderna. En la medida en que el enfoque científico es una protesta contra el pensamiento descuidado y el dogmatismo, 0 contra un providencialismo residual, hay mucho que decir al respecto. Pero muchas teorías en el molde clásico no están abiertas a este tipo de objeción. Los escritos de los grandes juristas internacionalistas de Victoria a Oppenheim (que, se puede argumentar, forman la base de la literatura tradicional del tema) son rigurosos y críticos. Hay abundancia de escritores contemporáneos que son lógicos y rigurosos en su enfoque y aún así no pertenecen a la escuela que he llamado científica: Raymond Aron, Stanley Hoffman y Kenneth Waltz son ejemplo de ello. Además, no resulta complicado encontrar casos en los que escritores del filón científico hayan fracasado en ser rigurosos y críticos en este sentido.

Mi séptima y última propuesta es que los practicantes del enfoque científico, al separarse de la historia y la filosofía, se han privado de los medios de autocrítica y, en consecuencia, tienen una visión de su tema y sus posibilidades que es inmadura y descarada. Deseo añadir que esto no es cierto, o no es igualmente cierto, de todos ellos. Pero su pensamiento está ciertamente caracterizado por una falta de cualquier sentido de indagación en la política internacional como una continua tradición de la que son los últimos reclutas; por una insensibilidad a las condiciones de la historia reciente que los han producido, les han proporcionado 
las preocupaciones y perspectivas que tienen, y los han coloreado de maneras que quizás no sean conscientes; por una ausencia de cualquier disposición para preguntarse por qué, si los frutos que prometen sus investigaciones son tan grandes y las perspectivas de traducirlos en acciones tan favorables, esto no lo ha logrado nadie antes; por una actitud acrítica hacia sus propios supuestos y, especialmente, hacia la actitud moral y política que tienen una posición central pero no reconocida en gran parte de lo que dicen.

El enfoque científico en las Relaciones Internacionales proporcionaría un tema muy adecuado para el tipo de crítica que Bernard Crick ha aplicado a un objetivo más amplio en su admirable libro The American Science of Politics - crítica que, al describir su historia y condiciones sociales, aislaría la subestructura esbelta y provinciana de la asunción moral y política que subyace a la empresa7-. Existe una pequeña duda de que la concepción de una ciencia de la política internacional, como el de una ciencia de la política en general, ha echado raíces y florecido en Estados Unidos debido a las actitudes hacia la práctica de los asuntos internacionales que son especialmente estadounidenses - supuestos, en particular acerca de la simpleza moral de los problemas de la política exterior, la existencia de "soluciones" a estos problemas, la receptividad de los policy-makers a los frutos de la investigación, y el grado de control y manipulación que puede ser ejercido en todo el campo diplomático por cualquier país-.

\section{III}

Habiendo planteado el caso en contra del enfoque científico, debo regresar a la calificación que introduje al principio. Soy consciente de haber atacado con una escopeta a toda una bandada de aproximaciones variadas, donde los disparos de un solo rifle podrían haber derribado a los objetivos principales de manera más eficiente y al mismo tiempo librado a otros que podrían haber resultado heridos innecesariamente. Ciertamente, hay muchos más enfoques para la teoría de las Relaciones Internacionales que dos, y la dicotomía que ha servido a mi propósito actual oculta muchas otras distinciones que es importante tener en cuenta.

Los estudiantes de Relaciones Internacionales están divididos por lo que en algunos casos son simplemente barreras de malentendidos o prejuicios académicos que atraviesan todo el campo de los estudios sociales en la actualidad. Sin duda, es deseable que esas barreras sean rebajadas. Con todo, en la presente controversia, el eclecticismo disfrazado de tolerancia, es el mayor peligro de todos; si vamos a ser hospitalarios con todos los enfoques (porque "algo se puede extraer de ellos algún día") y extender iguales derechos a todos los tópicos (porque "existe, después de todo, un ápice de verdad en lo que dice"), no habría final a las absurdidades empujadas sobre nosotros. Hay ápices de verdad que se pueden obtener de un orador en Hyde Park Corner o de un hombre en un autobús de Clapham, pero la pregunta es "¿qué lugar ocupan en la jerarquía de prioridades académicas?".

Espero haber dejado claro que veo una gran cantidad de mérito en varias de las con-

\footnotetext{
7 Crick, Bernard, The American Science of Politics: Its Origins and Conditions, University of California Press, Berkeley y Londres, 1959.
} 
tribuciones que han hecho los teóricos que adoptan un enfoque científico. El argumento no es que estas contribuciones son inútiles, sino que lo que tiene valor en ellas se puede acomodar fácilmente dentro del enfoque clásico. Además, el peculiar método y las aspiraciones que dichas teorías han traído al tema, lo están llevando por un camino falso, y ante todos los llamados para seguirlo debemos permanecer resueltamente sordos.

\section{Bibliografía}

Boulding, Kenneth, Conflict and Defense: A General Theory, Harper \& Brothers, Nueva York, 1962.

Crick, Bernard, The American Science of Politics: Its Origins and Conditions, University of California Press, Berkeley y Londres, 1959.

Deutsch, Karl, et al., Political Community and the North Atlantic Area: International Organization in the Light of Historical Experience, Princeton University Press, Princeton, 1957.

Deutsch, Karl, Political Community at the International Level, Princeton University Press, Nueva Jersey, 1953.

Kaplan, Morton A. "Problems of Theory Building and Theory Confirmation in International Politics" en World Politics, vol. 14, no. 1, 1961, pp. 6-24.

Kaplan, Morton A., System and Process in International Politics, John Wiley \& Sons, Nueva York, 1957. Modelski, George, A Theory of Foreign Policy, Praeger for the Center of International Studies, Princeton University, Nueva York, 1962.

Morgenstern, Oskar, The Question of National Defense, Random House, Nueva York, 1959.

Rapoport, Anatol, Fights, Games, and Debates, University of Michigan Press, Ann Arbor, 1960.

Richardson, Lewis, Arms and Insecurity: A Mathematical Study of the Causes and Origin of War, Boxwood Press, Pittsburg, 1960.

Riker, William, The Theory of Political Coalitions, Yale University Press, New Haven, 1962.

Russet, Bruce, Community and Contention: Britain and America in the Twentieth Century, Cambridge University Press, Cambridge, 1963.

Schelling, Thomas, The Strategy of Conflict, Harvard University Press, Cambridge, 1960.

Wright, Quincy y C.C. Lienau (eds.), Statistics of Deadly Quarrels, Boxwood Press, Pittsburg, 1960. 


\section{RELACIONES INTERNACIONALES}

Revista académica cuatrimestral de publicación electrónica Grupo de Estudios de Relaciones Internacionales (GERI) Universidad Autónoma de Madrid, España

www.relacionesinternacionales.info

ISSN 1699 - 3950

ff facebook.com/RelacionesInternacionales

twitter.com/RRInternacional 